\title{
FORMATION OF A TOXIC METABOLITE FROM GENTAMICIN BY A HEPATIC CYTOSOLIC FRACTION
}

\author{
Sherry A. Crann, May Y. Huang, John D. Mclaren and Jochen Schacht* \\ Department of Otolaryngology, Kresge Hearing Research Institute, University of Michigan, Ann \\ Arbor, MI 48109, U.S.A.
}

(Received 16 October 1991; accepted 17 December 1991)

\begin{abstract}
We have demonstrated recently that incubation of the aminoglycoside gentamicin with an hepatic post-mitochondrial fraction produces a compound toxic to sensory cells from the inner ear in short-term culture; in contrast, the parent aminoglycoside was non-toxic in vitro (Huang MY and Schacht J, Biochem Pharmacol 40: R11-R14, 1990). In the present study, we investigated the subcellular distribution of the enzymatic activity and the nature of the metabolite. Isolated outer hair cells from the guinea pig cochlea were used to assay for cytotoxicity. The enzyme(s) responsible for this novel reaction of aminoglycosides was exclusively localized to the cytosolic fraction of guinea pig liver. No activity was detected in nuclear, lysosomal/mitochondrial or microsomal preparations. Furthermore, the toxin-forming enzymatic activity was associated with the high molecular weight fraction of the cytosol and did not require low molecular weight components. Filtration of the toxin through molecular weight cut-off membranes showed a molecular size of approximately 500 . This evidence is consistent with the toxin being a gentamicin derivative.
\end{abstract}

Biotransformation of drugs by the liver, the major organ for xenobiotic metabolism, has been well established. The formation of reactive metabolites may indeed be the underlying mechanism of a variety of toxic drug actions. Aminoglycoside antibiotics (e.g. streptomycin, neomycin and gentamicin) are recovered from the body largely unchanged, and it has been assumed, therefore, that they do not undergo significant metabolism [1]. A radiotracer study [2] reported the presence of some nongentamicin material in the urine of subjects receiving tritiated gentamicin but the relation to the injected material was unknown. While the acute and reversible actions of aminoglycosides may not require metabolism [3], the expression of irreversible cytotoxicity appears to be linked to a metabolite rather than the parent drug. Aminoglycosides which damage the kidney and the inner ear in vivo $[4,5]$ are not cytotoxic for isolated cochlear outer hair cells or renal cells in vitro [6,7]. Earlier we presented evidence suggestive of a metabolic step involved in aminoglycoside toxicity [8] and more recently demonstrated the gentamicin-dependent formation of a cytotoxin [9] by a post-mitochondrial "S9" fraction $[10]$ in vitro.

An understanding of this novel reaction of aminoglycosides requires knowledge of the nature of the metabolite(s) and the enzyme(s) involved. Microsomal enzymes linked to cytochrome $\mathrm{P} 450$ are commonly associated with drug metabolism, but enzymes in other subcellular compartments will also modify xenobiotics. Some monooxygenase reactions are carried out by soluble enzymes [11] as are structural modifications such as acylation,

\footnotetext{
* Corresponding author: Dr. Jochen Schacht, Kresge Hearing Research Institute, University of Michigan, Ann Arbor, MI 48109-0506. Tel. (313) 763-3572; FAX (313) 747-0693.
}

methylation, and sulfation, as well as conjugation reactions with glucuronides and glutathione [12]. Enzymes of amine metabolism which may be relevant for modification of aminoglycosides are also found in nuclear and mitochondrial fractions.

In this study, we localized the enzyme(s) of gentamicin metabolism in subcellular fractions from guinea pig liver. In addition, we provide evidence that the toxin is a gentamicin derivative. The recently developed bioassay with isolated outer hair cells $[6,9]$ was employed to detect toxin formation.

\section{MATERIALS AND METHODS}

Subcellular fractionation. Liver from pigmented guinea pigs ( $200-250 \mathrm{~g}$ body weight) was minced and homogenized in 3 vol. of $250 \mathrm{mM}$ sucrose and $20 \mathrm{mM}$ sodium HEPES ( $N$-[2-hydroxyethyl] piperazine- $N^{\prime}$ [2-ethanesulfonic acid]), $\mathrm{pH} \mathrm{7.4,} \mathrm{in} \mathrm{a} \mathrm{teflon/glass}$ homogenizer for $30 \mathrm{sec}$ (two passes) with the pestle driven at approximately $1,000 \mathrm{rpm}$. The homogenate was filtered through gauze and centrifuged for $10 \mathrm{~min}$ at $1,500 \mathrm{~g}$ to obtain a nuclear pellet and a postnuclear supernatant. The latter was separated into a mitochondrial/lysosomal pellet and supernatant by centrifugation for $20 \mathrm{~min}$ at $12,000 \mathrm{~g}$. The postmitochondrial supernatant was centrifuged for $1 \mathrm{hr}$ at $100,000 \mathrm{~g}$ to yield a microsomal pellet and the cytosol. Each pellet was washed gently in homogenizing buffer and centrifuged again at the appropriate speed. The washed pellets were then resuspended to the volume of the original homogenate. For some experiments, cytosolic fractions were desalted by gel exclusion chromatography over Sephadex G-25 (Pharmacia, Uppsala, Sweden). All operations were carried out at $0-4^{\circ}$, and fractions were stored in glass tubes at $-20^{\circ}$.

Formation of metabolite. Aliquots of each fraction were incubated with (final concentrations) $100 \mathrm{mM}$ 
sodium phosphate buffer or $20 \mathrm{mM}$ sodium HEPES, pH 7.4, $4 \mathrm{mM}$ NADPH, $30 \mathrm{mM} \mathrm{KCl}, 7 \mathrm{mM} \mathrm{MgCl}$, and $10 \mathrm{mM}$ gentamicin sulfate (Sigma, St. Louis, MO) for $1 \mathrm{hr}$ at $37^{\circ}$. Reactions were terminated without inactivating the toxin by heating the mixtures for $1.5 \mathrm{~min}$ at $55^{\circ}$. Protein was removed by centrifugation at $12,000 \mathrm{~g}$ for $10 \mathrm{~min}$, and the deproteinated incubation mixture was filtered through $0.2 \mu \mathrm{m}$ filters (Acro LC13, Gelman Science, Ann Arbor, MI) and kept on ice for immediate toxicity assay or stored in glass tubes at $-20^{\circ}$ for no longer than 1 week.

Cytotoxicity assay. Outer hair cells were isolated from the apical cochlear turns of pigmented guinea pigs by microdissection [13]. Yield of viable cells from the apex by far exceeds that from lower turns making apical cells the preferred substrates in studies of isolated outer hair cells. The cells were maintained at room temperature in Hanks' Balanced Salt Solution (HBSS) without phenol red or sodium bicarbonate, buffered with $5 \mathrm{mM}$ sodium HEPES, $\mathrm{pH} 7.4$, and adjusted to $300 \pm 1 \mathrm{mOsM}$ with $\mathrm{NaCl}$. For cytotoxicity assays, the isolated cells were exposed for $60 \mathrm{~min}$ to the deproteinated incubation mixtures diluted 1:10 (v/v) in HBSS. Membrane integrity of cells was determined by exclusion of the nuclear dye propidium iodide (final concentration, $1 \mu \mathrm{M})$; stained, enucleated, granular, or severely swollen cells were considered non-viable.

Molecular size of metabolite. A molecular weight cut-off membrane (SpectraPor 100, 500, and 1000 from Spectrum, Los Angeles, CA; 12,000 from Sigma) was placed in a centrifugal filter apparatus (Rainin, Woburn, MA). The deproteinated reaction mixture containing the toxic metabolite was first passed through a $0.2 \mu \mathrm{m}$ syringe filter and then centrifuged at $19,200 \mathrm{~g}$ for $1-2 \mathrm{hr}$. After centrifugation, the membranes were examined under a microscope to ensure that no tears or holes were present. The filtrates were then assayed for cytotoxic activity.

Analysis and purification of gentamicin. Commercial gentamicin (gentamicin sulfate, Schering, Bloomfield, NJ) was analyzed by TLC. Samples were developed on HPTLC Silica Gel 60 (E. Merck, Gibbstown, NJ) in the lower phase of chloroform:methanol:29\% aqueous ammonia ( $1: 1: 1$, by vol.) [14]. TLC tanks were equilibrated with the solvent for several hours before each run. After development, the plates were dried and then sprayed with a solution of fluorescamine (Sigma) in acetone $(0.1 \mathrm{mg}$ fluorescamine $/ \mathrm{mL}$ acetone), and fluorescent bands were visualized under long-wave UV light. This analysis resolved the gentamicin components $C_{1}, C_{1 a}$ and $C_{2}$ as major bands and at least six minor amine-reactive compounds.

The major components $C_{1}, C_{1 a}$ and $C_{2}$ of commercial gentamicin were purified on Silica Gel 60 with a preadsorbent layer without fluorescence indicator (E. Merck). Five hundred microliters of an aqueous solution of $10 \mathrm{mg}$ gentamicin sulfate $/ \mathrm{mL}$ was spotted on the preadsorbent layer across the plate and allowed to dry. The developing solvent was the upper layer of chloroform: methanol:29\% aqueous ammonia $(1: 1: 1$, by vol.). After development, $1-\mathrm{cm}$ wide vertical strips along each edge and in the middle of the plate were sprayed successively with $1 \%$ triethylamine (Pierce Chemical, Rockford, IL) in acetone, fluorescamine (as described above), and triethylamine, waiting several minutes between each spray. The major components of gentamicin, detected under long-wave UV light, migrated with an $R_{f}>0.7$. After marking the position of gentamicin and removing the sprayed bands, the major gentamicin bands were scraped into a plastic centrifugation tube. Gentamicin was eluted by adding $5 \mathrm{~mL}$ of water, mixing repeatedly, and sedimenting the silica gel in a table-top centrifuge. The clear supernatant was removed, lyophilized, reconstituted into $300 \mu \mathrm{L}$ of HBSS, and cleared from undissolved material through a prewashed $0.45 \mu \mathrm{m}$ nylon centrifugal filter (Scientific Resources, Somerset, NJ). Analytical TLC of this preparation showed the presence of gentamicin $C_{1}, C_{1 a}$ and $C_{2}$ without contamination by amine-reactive compounds.

\section{RESULTS}

The "S9" fraction which we had used previously to metabolize gentamicin [9] is essentially a postmitochondrial supernatant. In preliminary experiments, a post-mitochondrial supernatant from guinea pig liver was further separated into a microsomal pellet and a cytosolic supernatant. The enzymatic activity that generated the cytotoxin in the presence of gentamicin remained with the cytosol, and little activity was found in the crude microsomes. The toxicity of the drug after incubation with the postmitochondrial supernatant $(3.6 \mathrm{mg}$ protein $/ \mathrm{mL}$ incubation) or the cytosol $(3.1 \mathrm{mg}$ protein $/ \mathrm{mL}$ incubation) was almost identical: percent viability of isolated outer hair cells was reduced to $24 \pm 13$ and $25 \pm 9$, respectively. Exposure of cells to control incubations without enzyme or without gentamicin consistently left $70-80 \%$ of the cells viable; this was similar to the viability of untreated cells immediately after isolation $[6,9]$.

Subsequently, toxin-generating enzymatic activity was quantified in all subcellular fractions (nuclei, mitochondria/lysosomes, microsomes, and cytosol) and compared to the activity in the total homogenate (Fig. 1). There was no significant formation of cytotoxin when gentamicin was incubated with the nuclear, mitochondrial/lysosomal, or microsomal fractions. Viability of hair cells remained between 70 and $80 \%$ when they were exposed to gentamicin treated with these fractions. In contrast, the cytosol produced a toxic metabolite. The toxin-generating potency of the cytosol was quantitatively similar to that of the original homogenate, indicating that essentially all of the activity was recovered. Cell viabilities were reduced to $28 \pm 2 \%$ or $20 \pm 4 \%$ after exposure to gentamicin metabolized with homogenate or cytosol, respectively.

Homogenate and cytosol contain both high and low molecular weight components. To assess a possible contribution of endogenous small compounds to gentamicin metabolism, low molecular size components were removed from the cytosol by chromatography over Sephadex G-25 (Table 1). The activity producing the cytotoxin was unaffected and remained associated with the high molecular size 


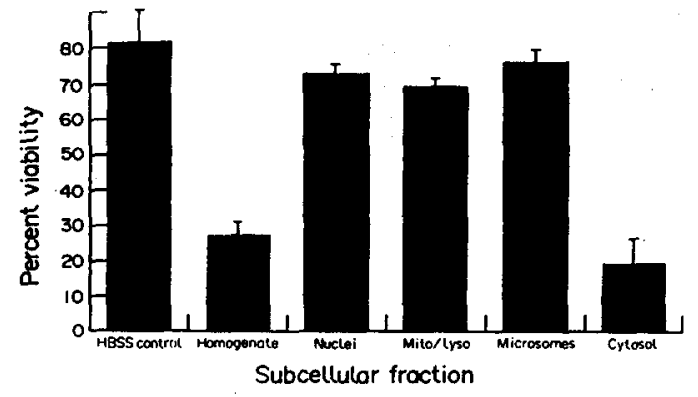

Fig. 1. Subcellular distribution of gemtamicin-metabolizing enzyme activity. Gentamicin was metabolized with the subcellular fractions and cytotoxicity assayed as described in Materials and Methods. All fractions were added to the incubations in aliquots whose volume was identical to that of the homogenate. The final protein concentration per milliliter of incubation medium was $5.6 \mathrm{mg}$ for homogenate, $0.9 \mathrm{mg}$ for nuclei, $0.6 \mathrm{mg}$ for mitochondria/lysosomes, $0.3 \mathrm{mg}$ for microsomes, and $3.2 \mathrm{mg}$ for cytosol. Percent viability is given as the mean $\pm \mathrm{SD}(\mathrm{N}=3)$. The activity in the homogenate and the cytosol was significantly different from that in the other fractions at $\mathrm{P}<0.001$ (Student's $t$ test).

eluate $(>5,000 \mathrm{Da})$. Similarly, exhaustive dialysis and treatment of cytosol with activated charcoal for the removal of low molecular size compounds left the enzymatic activity essentially unchanged (data not shown). Toxin formation proceeded even in the absence of NADPH, indicating that no exogenous cofactors were necessary for the reaction (Table 1). As observed before, omission of gentamicin or the cytosol from the incubations precluded generation of a toxin.

In contrast to the enzymatic activity required for metabolism, the toxin itself exhibited an apparent molecular size of between 10 and 1,000 Da (Table
Table 2. Molecular size of the cytotoxic metabolite

\begin{tabular}{lcc}
\hline $\begin{array}{l}\text { Molecular size } \\
\text { of filtrate }\end{array}$ & $\begin{array}{c}\text { Cells } \\
\text { observed }\end{array}$ & $\begin{array}{c}\text { Viable cells } \\
\text { (\% of total) }\end{array}$ \\
\hline Unfiltered cytosol & 350 & 26 \\
$<12,000$ & 184 & $27^{*}$ \\
$<1,000$ & 356 & $37^{*}$ \\
$<500$ & 171 & $50 \dagger$ \\
$<100$ & 240 & $76 \dagger$ \\
Control: no cytosol & 369 & 72
\end{tabular}

Gentamicin was metabolized with a cytosolic fraction as described in Materials and Methods and the cytotoxin filtered through membranes with the permeation limits listed above. The results represent two to four independent experiments, and calculations of viability were based on the total number of cells observed. Statistical significance was determined by ANOVA of individual experiments.

* Differs significantly $(\mathrm{P}<0.05)$ from control but not from unfiltered cytosol.

$\dagger$ Differs significantly $(\mathrm{P}<0.05)$ from unfiltered cytosol but not from control.

2). It passed through molecular size cut-off membranes of 12,000 and $1,000 \mathrm{Da}$; these filtrates were as toxic as the unfiltered control. Filtrate through the $100 \mathrm{Da}$ membrane had lost its toxicity, indicating that the toxin was retained at this exclusion limit. The $500 \mathrm{Da}$ fraction exhibited intermediate toxicity.

Commercial gentamicin consistently showed minor amine-reactive contaminants upon chromatographic analysis in addition to the three major isomers $C_{1}$, $\mathrm{C}_{1 \mathrm{a}}$ and $\mathrm{C}_{2}$. However, gentamicin purified by thinlayer chromatography still produced a toxin (Table 3 ) of apparently unchanged activity.

\section{DISCUSSION}

Drugs and xenobiotics are frequently metabolized

Table 1. Association of gentamicin-metabolizing activity with the high molecular size fraction of cytosol

\begin{tabular}{lcc}
\hline Fraction & $\begin{array}{c}\text { Cells observed } \\
\text { (total number) }\end{array}$ & $\begin{array}{c}\text { Viable cells* } \\
\text { (\% of total) }\end{array}$ \\
\hline Complete cytosol & 399 & 35 \\
Desalted cytosol & 1192 & 37 \\
Desalted cytosol - NADPH & 456 & 41 \\
Control: cytosol omitted & 352 & 72 \\
Control: gentamicin omitted & 212 & 68 \\
\hline
\end{tabular}

Gentamicin was metabolized and cytotoxicity assayed as described in Materials and Methods. The results represent combined data from three to seven assays per condition in three independent experiments. For each experiment, a desalted cytosol was prepared (protein concentration, 2.0 to $2.8 \mathrm{mg} / \mathrm{mL}$ incubation medium) and compared to its corresponding complete cytosol (protein concentration, 1.8 to $2.8 \mathrm{mg} / \mathrm{mL}$ incubation medium).

* Statistical significance was determined from individual experiments by a $t$ test: complete cytosol vs controls: $\mathbf{P}<0.01$; desalted cytosol vs controls: $P<0.01$; complete vs desalted cytosol: not significant; desalted cytosol with vs without NADPH: not significant; and control without cytosol vs control without gentamicin: not significant. 
Table 3. Formation of a cytotoxic metabolite from purified gentamicin

\begin{tabular}{lcc}
\hline Preparation & $\begin{array}{c}\text { Cells observed } \\
\text { (total number) }\end{array}$ & $\begin{array}{c}\text { Viable cells } \\
\text { (\% of total) }\end{array}$ \\
\hline Commercial gentamicin & 254 & 35 \\
Purified gentamicin & 259 & 44 \\
Control: HBSS & 166 & 75 \\
\hline
\end{tabular}

Gentamicin was purified and metabolized, and cytotoxicity was assayed as described in Materials and Methods. The results represent combined data from two assays per preparation.

in vivo by one of two general classes of reactions. One metabolic strategy generates a more polar metabolite from the parent compound, facilitating its renal excretion. These reactions are usually associated with microsomal cytochrome P450s, monamine oxidases and monooxygenases [12]. In contrast, reactions rendering a compound more hydrophobic, such as methylations, ethylations or acylations, are carried out by soluble enzymes [15]. Moreover, several enzymes of polyamine metabolism also are cytosolic. The latter are interesting candidates for an enzymatic conversion of aminoglycosides in view of structural and functional similarities between polyamines and aminoglycosides.

Our results clearly indicate that the enzyme(s) of gentamicin metabolism is soluble. Throughout the subcellular fractionation, the enzymatic activity remained associated with the soluble fractions, and no loss of activity was observed. The fact that NADPH was not essential for the formation of the toxin is in contrast to our preliminary study [9] where a dependence on pyridine nucleotides suggested the involvement of P450-linked enzymes. The conditions of the two studies are sufficiently different (use of a hepatic post-mitochondrial "S9" fraction from phenobarbital-treated animals vs a cytosolic fraction from untreated animals) to preclude direct comparisons or to provide an explanation for the discrepancy. It now seems clear, however, that a cytosolic enzyme (or enzymes) without apparent cofactor requirement can account for the metabolism of gentamicin to a toxic metabolite.

The identity of the metabolite remains speculative. Our original findings [9] could not rule out a gentamicin-dependent formation of a cytotoxin from an endogenous precursor in the " $\mathrm{S} 9$ " fraction. The present results suggest that the toxin is derived from gentamicin. The toxin has an apparent molecular size near 500 which corresponds well to that of the gentamicin complex (range' for gentamicins $\mathrm{C}_{1}, \mathrm{C}_{1 \mathrm{a}}$ and $C_{2}: 454$ to 482 ). Moreover, the high molecular size fraction of the cytosol containing the toxinforming enzymatic activity should be devoid of significant amounts of potential small endogenous precursors after Sephadex G-25 exclusion chromatography, exhaustive dialysis or treatment with activated charcoal.

One final uncertainty about the nature of the toxin was raised by the presence of amine-reactive impurities in commercial gentamicin. The fact that purified gentamicin still produced a toxin apparently excludes the possibility that such contaminants rather than the major components $\mathrm{C}_{1}, \mathrm{C}_{1 \mathrm{a}}$ and $\mathrm{C}_{2}$ give rise to the cytotoxin. This notion is further supported by preliminary experiments showing the formation of toxic metabolites from other aminoglycosides, tobramycin and kanamycin [16].

An important question to be addressed is the relationship between our in vitro observations and the in vivo toxicity of aminoglycosides. Several potential extracellular and intracellular sites of drug action have been established [3]. The acute and reversible effects of aminoglycosides can be explained by the displacement of calcium ions from the plasma membrane and the block of voltage-gated calcium channels of outer hair cells [6]. This may be an action in addition to a block of transduction channels which has been shown to occur with streptomycin in the hair cells of the bullfrog saccule [17]. On the cytoplasmic side of the plasma membrane, phosphatidylinositol-4,5-bisphosphate acts as a "receptor" for aminoglycosides $[3,18]$. Inhibition of phosphoinositide metabolism should interfere with the generation of second messengers from the lipid and thus with essential mechanisms of cell physiology. To what extent these actions of the native aminoglycosides contribute to the chronic toxicity remains to be established but none of these actions has been linked directly to irreversible damage to hair cells. Such irreversible damage may thus require the presence of the cytotoxic metabolite. This is consistent with observations that aminoglycosides can be detected in the inner ear and in hair cells long before physiological or structural damage is observed $[19,20]$. Thus, the formation of the metabolite or its accumulation to critical concentrations appears to be a rate-limiting step in the ioxic reaction sequence.

The extent of metabolism of aminoglycosides in vivo-both in terms of its rate and localization-is unknown. It is conceivable that the concentration of the metabolite is relatively low, making it a highly toxic species. This would explain why a toxic aminoglycoside metabolite has thus far escaped traditional methods of detection. Although the liver is capable of producing the metabolite, we cannot assume that this is the sole source or even one source of the metabolite for the destruction of kidney and inner ear. The liver is the major organ for drug metabolism in the body, but it is known that other tissues contribute to drug metabolism [21]. In fact, a local contribution to the development of organspecific toxicity of aminoglycosides is suggested by reports that aminoglycosides applied directly into the fluids of the inner ear in vivo [22] or to otocyst whole organ cultures [23] produce toxic effects. The tissue distribution of drug-metabolizing enzymes is generally not uniform throughout the body [21] and several organs, including the inner ear, seem capable of aminoglycoside metabolism (unpublished observations). We therefore can postulate a higher endowment of the enzymes of aminoglycoside metabolism in renal and inner ear tissues to explain selective tissue damage. Similarly, a differential 
distribution of these enzymes between the base and apex of the cochlea could explain the fact that the base is more sensitive to aminoglycosides than the apex.

On the other hand, tissue sensitivity to aminoglycosides may reside in detoxification mechanisms rather than in the production of the cytotoxin alone. Liver which is not damaged by aminoglycosides is also a major organ for drug detoxification by mechanisms based on glucuronidation or conjugation with sulfhydryl compounds [12]. Toxic metabolites generated in the liver may be detoxified and only reach low steady-state concentrations. Glutathionerelated pathways have been implicated in the detoxification of kanamycin $[24,25]$, and the inactivation of the metabolite by sulfhydryl compounds and radical scavengers can be demonstrated in vitro [26]. Thus susceptibility may depend not only on the potential for forming the metabolite but also on the ability of tissues to inactivate it.

The enzyme(s) and conditions of aminoglycoside metabolism now need to be further characterized. It may be that the metabolite is generated by a known enzymatic reaction hitherto not expected to use aminoglycosides as substrates. The finding of a previously undetected cytotoxic metabolite(s) of gentamicin offers a new approach to defining the mechanism of aminoglycoside toxicity, and to devising preventive strategies.

Acknowledgements-The authors wish to thank Ms. Loretta Hammel for her contributions to this study. This work was supported by Research Grant DC 00124 and Training Grant DC 00024 from the National Institutes of Health.

\section{REFERENCES}

1. Sande MA and Mandell GL, Antimicrobial agents. The aminoglycosides. In: The Pharmacological Basis of Therapeutics (Eds. Goodman AG, Gilman A, Rall TW and Murad F), 7th Edn, pp. 1150-1169. Macmillan, New York, 1985.

2. Wilson TW, Mahon WA, Inaba T, Johnson GE and Kadar D, Elimination of tritiated gentamicin in normal human subjects and in patients with severely impaired renal function. Clin Pharmacol Ther 14: 815-822, 1973

3. Schacht J, Molecular mechanisms of drug-induced hearing loss. Hear Res 22: 297-304, 1986.

4. Hawkins JE, Drug ototoxicity. In: Handbook of Sensory Physiology (Eds. Keidel WD and Neff WD), pp. 707-748. Springer, New York, 1976.

5. Whelton A, Treatment of gram-negative infections in patients with renal impairment: New alternatives to aminoglycosides. J Clin Pharmacol 28: 866-878, 1988.

6. Dulon D, Zajic G, Aran J-M and Schacht J, Aminoglycoside antibiotics impair calcium-entry but not viability and motility of cochlear outer hair cells. J Neurosci Res 24: 338-346, 1989.

7. Hori R, Yamamoto $K$, Saito $H$, Kohno $M$ and Inui $\mathrm{KI}$, Effect of aminoglycoside antibiotics on cellular functions of kidney epithelial cell line (LLC-PK $\left.)_{1}\right)$ : A model system for aminoglycoside nephrotoxicity. $J$ Pharmacol Exp Ther 230: 742-748, 1984.

8. Takada A, Bledsoe $S$ and Schacht $J$, An energydependent step in aminoglycoside ototoxicity: Prevention of gentamicin ototoxicity during reduced endolymphatic potential. Hear Res 19: 245-251, 1985.

9. Huang MY and Schacht J, Formation of a cytotoxic metabolite from gentamicin by liver. Biochem Pharmacol 40: R11-R14, 1990.

10. Maron DM and Ames BN, Revised methods for the Salmonella mutagenicity test. In: Handbook of Mutagenicity Test Procedures (Eds. Kilbey BJ, Legator M, Nichols W and Ramel C), pp. 93-140. Elsevier Science Publishers, Amsterdam, 1984.

11. Gelboin HV and Friedman FK, Monoclonal antibodies for studies on xenobiotic and endobiotic metabolism. Biochem Pharmacol 34: 2225-2234, 1985.

12. Nelson SD and Gordon WP, Mammalian drug metabolism. J Nat Prod 46: 71-78, 1983.

13. Zajic $G$ and Schacht J, Comparison of isolated outer hair cells from five mammalian species. Hear Res 26 : 249-256, 1987

14. Sekkat $M$, Fabre $H$, Simeon De Buochberg $M$ and Mandrou B, Determination of aminoglycosides in pharmaceutical formulations-I. Thin-layer chromatography. J Pharm Biomed Anal 7: 883-892, 1989.

15. Weinshilboum $\mathbf{R}$, Pharmacogenetics of methylation: Relationship to drug metabolism. Clin Biochem 21: 201-210, 1988.

16. Huang MY and Schacht J, Non-microsomal metabolites of aminoglycosides are cytotoxic to cochlear outer hair cells. Otolaryngol Head Neck Surg 105: 191, 1991.

17. Kroese A, Das A and Hudspeth A, Blockage of the transduction channels of hair cells in the bullfrog sacculus by aminoglycoside antibiotics. Hear Res 37: 203-217, 1989.

18. Schacht J, Isolation of an aminoglycoside receptor from guinea pig inner ear tissues and kidney. Arch Otorhinolaryngol 224: 129-134, 1979.

19. Tran Ba Huy P, Bernard P and Schacht J, Kinetics of gentamicin uptake and release in the rat: Comparison of inner ear tissues and fluids with other organs. J Clin Invest 77: 1492-1500, 1986.

20. Hiel H, Ph.D. Thesis. University of Bordeaux, Bordeaux, France, 1991.

21. Ziegler DM, Flavin-containing monooxygenases: Catalytic mechanism and substrate specificities. Drug Metab Rev 19: 1-32, 1988.

22. Takada $\mathbf{A}$ and Schacht J, Calcium antagonism and reversibility of gentamicin-induced loss of cochlear microphonics in the guinea pig. Hear Res 8: 179-186, 1982.

23. Anniko M, Takada A and Schacht J, Comparative ototoxicities of gemtamicin and netilmicin in three model systems. Am J Otolaryngol 3: 422-433, 1982.

24. Hoffman DW, Whitworth CA, Jones KL and Rybak LP, Nutritional status, glutathione levels, and ototoxicity of loop diurectics and aminoglycoside antibiotics. Hear Res 31: 217-222, 1987.

25. Hoffman DW, Whitworth CA, Jones-King $\mathrm{KL}$ and Rybak LP, Potentiation of ototoxicity by glutathione depletion. Ann Otol Rhinol Laryngol 97: 36-41, 1988.

26. Garetz $S$ and Schacht J, Sulfhydryl compounds reduce gentamicin-induced outer hair cell damage in vitro. Abstr Assoc Res Otolaryngol 15: 110, 1992. 